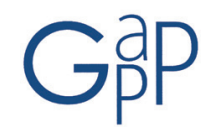

GESTIÓN Y ANÁLISIS DE POLÍTICAS PÚBLICAS, Nueva Época, nº 12 julio-diciembre 2014 ISSN: 1989-8991

DOI: http://dx.doi.org/10.24965/gapp.voi12.10208

\title{
Evolución de modelos y enfoques para el análisis de la "acción pública": del discurso a una nueva dinámica normativa
}

\author{
Javier Pinazo Hernandis \\ Universidad CEU. Cardenal Herrera de Valencia \\ jpinazo@uch.ceu.es \\ Recibido: 27 de Octubre de 2014 \\ Aceptado: 10 de noviembre 2014
}

\begin{abstract}
Resumen
El análisis de la acción pública en los diversos niveles político-administrativos españoles parece no superar el carácter selectivo, sectorial o anecdótico, cuando no se queda en un mero discurso político.

La evolución de los modelos y enfoques analíticos de políticas reflejan posiciones muy distintas y a la par la gran dificultad de llevarlos a cabo, lo que invita a pensar que estamos ante otro discurso, esta vez científico-académico.

De otra parte los análisis teóricos cuando se utilizan son fundamentalmente descriptivos y generalmente reflejan los factores del juego político-institucional que impiden que se hagan de manera óptima o mejorable, y pocas veces cómo podrían mejorarse. A su vez los modelos teóricos normativos son de difícil o complejísima factura.
\end{abstract}

¿Es posible romper con esta dinámica o hay que empezar a buscar nuevos instrumentos analíticos?

Palabras clave

Acción pública, Análisis de políticas, Cross modern, Deconstrucción Político-Administrativa, Discurso, Normatividad.

\section{Evolution of models and approaches to the analysis of "public action": from speech to dynamic new rules}

Abstract

The analysis of public action in the various Spanish political and administrative levels seems not exceed selective, sectorial or anecdotal, when it is not merely a political speech.

The development of analytical models and policy approaches reflect very different positions and at par the great difficulty of carrying them out, which invites us to think that this is another speech, this time scientific-academic.

Furthermore the theoretical analysis when used are mainly descriptive and generally reflect the political and institutional factors play that prevent or improvable make optimal way, and rarely how they could be improved. In turn normative theoretical models are difficult or very complex bill.

Is it possible to break this dynamic or have to start looking for new analytical tools?

Keywords

Government Action, Policy Management, Cross Modern, Administrative and Political Deconstruction, Discourse, Normativity 


\section{1. -INTRODUCCIÓN: EL ANÁLISIS DE LA ACCIÓN PÚBLICA}

El estudio de la acción pública de los gobiernos democráticos, denominada en un primer momento como Ciencia de las Políticas, surge como "discurso científico" en 1951 con el trabajo La orientación hacia las políticas de H. Lasswell', seguido por el texto de C. Lindblom La Ciencia de salir del paso en 1959; T. Lowi, con Políticas Públicas, estudios de caso y teoría política de 1964²; A. Etzioni, que en 1967 publicaría La exploración combinada: un tercer enfoque de la toma de Decisiones; Y. Dror haría lo propio con Reexamen del Proceso de Políticas Públicas en 1968 y Prolegómenos para las Ciencias de Políticas en 1970. Con Esencia de la decisión de G. Allison en 1971 y T. R. Dye con Entendiendo Políticas Públicas en 1975 terminan prácticamente las obras más representativas y seminales de estos autores de primera generación ${ }^{3}$.

Ya en los primeros trabajos se cuestionaba si se trataba de realidad o de un mero discurso -político y científicodel análisis de políticas, en cuanto a si éstas determinan la política ${ }^{4}$ o lo hacía la economía ${ }^{5}$, cuestión que sigue patente en la literatura actual (Cejudo, 2008, Nateras, 2006).

El "discurso político" de las Políticas se introdujo en la campaña presidencialista de Robert F. Kennedy en 1968, al expresar (...) no vengo a luchar contra otros políticos (Macarty) sino a proponer otra manera de "hacer políticas"(...). Discurso precedido por su introducción de la evaluación de políticas educativas siendo senador por Nueva York en los años anteriores ${ }^{6}$.

En España se ha dicho que ya existen instituciones que realizan evaluaciones, bien en ámbitos sectoriales (ANECA, ANEP...) o en relación con la fiscalización del gasto público (Tribunal de Cuentas, IGAE. . . ) otrosí del cometido de la AEVAL (Garde, 2004:21-27).

El análisis o evaluación de la acción pública -que entendemos hic et nunc como equivalentes- sustentado en el análisis de políticas, tiene por objeto investigar si los medios jurídicos, administrativos y financieros aplicados permiten lograr los efectos esperados de dichas políticas y la consecución de los objetivos asignados? Es una actividad institucional que tiene vocación de integrarse en la Gestión Pública y en el funcionamiento del sistema político (Garde, 2006:17). La evaluación supone conocer para mejorar la acción (Gascó, 2002:55) y aunque la mayoría de las veces se identifica la evaluación con una acción a posteriori, para algún sector doctrinal también puede realizarse tanto en el diagnóstico, como en la fase de programación y se centra en los objetivos de la política o programa y a la par en las necesidades de los usuarios o beneficiarios (Aguilar y Ander-Egge, 1992).

Hay institucionalización de la evaluación cuando en el proceso político se legitima un marco normativo adecuado para el desarrollo de la evaluación y con la inserción de estructuras e instancias en los distintos niveles político-administrativos de gobierno y según las distintas actividades o sectores a evaluar. Conlleva la incorporación de la evaluación y sus resultados como prioridad de la agenda gubernamental y en los procesos de formación de políticas públicas y exige un modelo analítico permanente de resultados, conforme a principios y valores sustentadores (Garde, 2006:17).

Por último la metaevaluación es una investigación sistemática cuyo objetivo es emitir un juicio acerca de la calidad o los méritos de una evaluación, convirtiéndose ésta, a su vez, en el objeto de estudio del metaevaluador. El objetivo de la metaevaluación es asegurar la calidad de los servicios evaluativos, evitar las prácticas fraudulentas, desviadas o contrarias al interés público y señalar el camino para el perfeccionamiento de la empresa evaluativa (García, 2009). La metaevaluación, al igual que la evaluación es, en esencia, una actividad pluridisciplinar (Alvira,

1 Laswell publicó en 1971 otro gran referente: La concepción emergente de las Ciencias de Políticas.

2 Completado con Lowi, 1972, en el que añadiría las políticas constitutivas a su primera clasificación de políticas regulatorias, distributivas y redistributivas.

3 No obstante desde los 50 ha habido exposiciones muy válidas con avances sustanciales sobre los marcos teóricos, aparte de las propias reformulaciones de los autores pioneros. Entre ellos vg. Landau, Forester, Dahl, Majone, Meltsner, March y Olsen, Behn...

En España el primer rastro de discurso científico que hemos encontrado se remonta a la Ciencia administrativa de los "60 del S. XX. de la mano de Segismundo Royo-Vilanova al afirmar que (...) La política de la función pública es esencial para una reforma de la Administración encaminada para aumentar su rendimiento y eficacia... una política de la función pública, (que debe manifestarse en un Estatuto de funcionarios) puede repercutir favorable o desfavorablemente en la marcha de los servicios públicos(...). Conferencia a instancia del IEP en la sede de Fomento del Trabajo de Barcelona en mayo-abril de 1960 (Royo-Vilanova, 1961:58)

4 “Does policies matter?”, Lowi, 1972:299; Newton-Sharpe, 1983.

5 Dye, 1966.

6 Ya el Secretario de defensa USA (1961-1968) MacNamara había sido presentado como el precursor del análisis sistemático en las políticas públicas, desde su experiencia de analista en la USAF.

7 Según expresión del Consejo científico de evaluación francés - Conseil National de l’Evaluation (CNE)- 
1991) por lo que ninguna disciplina científica puede arrogarse la pretensión de convertirse en la "ciencia evaluadora" por excelencia ${ }^{8}$.

Cabe pues preguntarse si los modelos y enfoques propuestos permiten avanzar hacia una dinámica analítica normativa, capaz de salir tanto del discurso científico como del político y que sea también aplicativa y prescriptiva. Parece que entre el mero discurso y la metaevaluación hay un recorrido científico y político que hay que emprender en este escenario combinado de Cross Modern y de deconstrucción político-administrativa9 que sobrepasa a la arquitectura institucional.

\section{2. - EVOLUCIÓN DE LA DISCIPLINA}

\section{1 Las Ciencias de Políticas}

Las Ciencias de Políticas (Policy Sciences) en la propuesta original de Lasswell eran algo mucho más ambicioso que el Análisis de políticas (Policy Analysis) que fue la parte de las mismas que logró desarrollarse. En las Ciencias de Políticas se incluían las ciencias sociales que proveían inputs relevantes para las decisiones de gobierno, de negocios o sociales. Sus objetos eran tanto los métodos de investigación del proceso de la política como los resultados de los estudios de las políticas y los descubrimientos de las disciplinas que podían aportar contribuciones importantes para las necesidades de inteligencia del momento (Laswell, 1951)

Esto supone una orientación hacia las políticas (Policy Orientation) que contenga una doble dimensión del conocimiento "del" proceso de la política y "en" el proceso de la política". De una parte interesa el proceso de la política y, por otra, las necesidades de inteligencia de este proceso. En cuanto al conocimiento "del" proceso de la política busca desarrollar la ciencia de la formación y ejecución de las políticas, utilizando los métodos de investigación de las ciencias sociales y de la psicología.

El conocimiento "de" hace referencia al análisis y al estudio del proceso con pretensión cognoscitiva. "Knowledge of" alude a la tarea de conocer el proceso de decisión de la política, tal y cómo de hecho sucede, referido pues a la parte teórica.

El conocimiento "en" busca mejorar el contenido concreto de la información y de la interpretación disponibles para operadores de las políticas y, por consiguiente, rebasa las fronteras de las ciencias sociales. "Knowledge in" comprende la tarea de corregir y mejorar la decisión pública desde los postulados de las ciencias sociales (ciencias de decisión y gestión, métodos analíticos, inteligencia de la información...) con el propósito de incrementar la racionalidad de la acción política y con un carácter práctico y aplicativo.

Dror de su parte propuso una nueva Ciencia de políticas con un paradigma superador del de Laswell que habría de integrar las diversas ciencias sociales y las disciplinas de la decisión, enfocada a la elaboración de políticas y al estudio de la metapolítica, la política de las políticas (Dror, 1968).

\section{2 El Análisis de Políticas}

Para Lasswell la actividad de conocimiento "de" y "en" eran interdependientes, complementarias e interdisciplinarias y contextuales, con especial atención del contexto histórico y sociopolítico ambiental. Pero esta tendencia unitaria primigenia tendría dos reacciones en el mundo académico de su tiempo, configurándose así dos corrientes cuyas características más notorias fueron (Garson, 1986:149 y ss; Aguilar, 1999b:54 y ss):

a) Los interesados en el "conocimiento de" o sinópticos (que adoptaron la expresión Policy analysis) se caracterizaron por interesarse sólo en el contenido y en el momento normativo-técnico, o sea, en el análisis y diseño de la política. Eran normativos, cuantitativos, unidisciplinarios, economicistas, positivistas, tecnócratas, reemplazaban la política por el conocimiento, acentuaban la especulación intelectual, eran comprehensivos, planificadores, maximizadores, partidarios del análisis racional (sinóptico o incremental) y se identifican con el análisis de sistemas como metateoría, el empirismo estadístico como metodología y la optimización de valores como criterio de decisión.

b) Los interesados en el "conocimiento en" o antisinópticos (que adoptaron la expresión Policy Sciences), por el contrario se interesaron en el proceso decisorio, para principalmente entender cómo se tomaban de hecho las decisiones. Eran contextualizadores, multidisciplinarios, postpositivistas, defendían una faceta de político-

8 Véase que en la muy reciente crisis del ébola, Obama ha designado a Ron Klain, abogado y jefe de gabinete de dos ex vicepresidentes, experto en políticas públicas y gestión de crisis para coordinar las acciones gubernamentales y no a un experto en sanidad.

9 Pinazo, 2013:58. 
administradores, reemplazando el conocimiento por la política, pluralistas (ajuste mutuo partidario), conductistas, insistían en la interacción social, identificaban el pluralismo como metateoría, el análisis contextual y de casos como metodología y la racionalidad social como criterio de decisión.

Fue la tradición antisinóptica la dominante en la ciencia política, mientras que la sinóptica fue la ampliamente utilizada en la Administración Pública y en el análisis de políticas.

\section{3 Recepción en la Ciencia de la Administración}

La Ciencia Política ha estudiado sistemáticamente casi todo menos la manera de cómo el gobierno construye y desarrolla sus decisiones, ha prestado gran atención al sistema político y poco a sus productos (Aguilar, 1992b). Por su parte la Teoría de la Administración Pública ha quedado constreñida entre las consideraciones jurídicas e institucionales y/o algunas de carácter operativo. Al acercar la Administración al proceso de políticas no sólo volvemos a encontrarnos con los enfoques seminales en el sentido clásico de buen gobierno/buena administración, sino que podemos reconstruir la visión integral y sistémica de su objeto de estudio.

El auge académico de las políticas en la época conductista de los 70 respondió a la doble exigencia de encontrar respuestas en la Ciencia Política ante los problemas sociales del momento, a la par, superar la dicotomía entre política y administración. Se afirmó entonces que había sido excesiva la atención dada a la política como conjunto de actores, instituciones (inputs), en detrimento de la política como resultados (outputs). En la Ciencia administrativa se propuso abandonar tanto a la administración científica y el posterior POSDCORB ${ }^{10}$ para redirigir el locus y focus disciplinario en torno al proceso de la política pública (Frederickson, 1971).

La obra Implementación de Pressman- Vildawski, pionera en la subárea de la implementación de políticas (Implementation Research), lleva el provocador subtítulo de "Como las grandes esperanzas de Washington son destrozadas en Oakland o por qué es sorprendente que los programas federales pueden llegar a funcionar aunque sólo sea un poco". Éste refleja los problemas en la fase de ejecución, las notables fallas operativas y el gap entre el diseño y los actos de implementación, en definitiva, la diferencia entre lo que se diseña y lo que finalmente se ejecuta, lo que suponía una labor más propia del momento administrativo. Para los autores, "implementar" significa ejecutar, realizar, completar, producir... Sin embargo, algunos programas fracasaban por falta de acuerdo político, falta de fondos, desacuerdo entre los funcionarios locales y las empresas contratistas, y porque en el trascurso de la acción las condiciones iniciales de la política se alteran por una cadena de causalidades.

El estudio de la implementación exige comprender que la secuencia aparentemente sencilla de acontecimientos depende de complejas cadenas de interacción recíproca en la que no se puede aislar la política, ni puede ser relegada a debates independientes. Incluso deberían ser vistas con una mayor correspondencia mutua (PressmanVildawski, 1973).

La función principal del concreto análisis de la implementación consistiría en identificar los factores que condicionan el logro de los objetivos normativos a lo largo de todo el proceso, tales como los objetivos del programa, su capacidad para estructurar apropiadamente la ejecución, o el efecto de las variables políticas en el apoyo al programa (Sabatier-Mazmanian, 1981). En la puesta en práctica, el énfasis en el análisis reside en los aspectos causales de los éxitos o fracasos, y en la de los factores que condicionan el rendimiento político (Subirats, 1994:103-104)".

Antes de la obra pionera todo giraba en torno a la formulación (Gunn, 1978: 169-176), una vez surgido el interés por la implementación como un subproducto del interés por la esencia de la política en la era postconductivista, los trabajos efectuados contribuirían a la teoría explicativa y predictiva, no obstante la precaución de no saber muy bien el inicio y el fin de la fase de implementación que es objeto estudio análisis (Ingram, 1999:591-592).

Lo que diferencia este análisis es que el interés fundamental se centra más en las consecuencias de las políticas que en las estructuras y procesos, toda vez que se va diluyendo entre los estudiosos la sensación del fracaso general del análisis de políticas, al poder estudiarse con más detenimiento la relación causa/efecto de los factores determinantes (Ingram, 1999:610).

Puede pecarse también de un cierto maximalismo analítico en la fase de implementación para quienes estén más interesados en el (sub)sistema administrativo que en el político, por entender que casi la totalidad de los

10 ¿Cuáles son los elementos funcionales de la obra de un director ejecutivo se preguntaría? POSDCORB es la respuesta y su elementos son los siguientes: (P) Planificación, (S) dotación de personal, (D) dirección, (C) coordinación, (R) presentación de informes y (B) elaboración de presupuestos .) (Gülick, 1937). Representa el paradigma neoclásico de Administración, hasta la irrupción de las escuelas humanistas.

11 Otros enfoques puede verse en Smith, 1973; Van Meter-Van Horn, 1975; Rein-Rrabinovitz, 1978; Sabatier-Mazmanian, 1981. 
errores están en la praxis política, cuando la mayor o menor artificiosa separación política/ Administración-clave del sistema de Administración Pública clásica- aconseja un enfoque sistémico y funcional de la política y las políticas ${ }^{12}$, sin perjuicio de la instrumentalidad del proceso de fases desde otra no menos forzada lectura como ciclo vital.

G. Frederickson fue una de las primeras voces en denunciar'3 las fallas del sistema administrativo clásico, proponiendo una "Nueva Administración Pública (NAP)" que respondiera a preguntas tales ¿cómo podemos ofrecer más o mejores servicios con los recursos disponibles? (eficiencia), o ¿cómo podemos mantener nuestro nivel de servicios mientras gastamos menos dinero? (economía)?

En la perspectiva de Política Pública, la Administración Pública ni se considera una subárea de la Ciencia Política, ni tampoco la abarca y comprende. Se concibe simplemente como un sinónimo (Landau, 1972). Es decir la Gestión Pública mediante las políticas busca exactamente lo mismo tanto desde la perspectiva política como técnica: conformar la sociedad normativamente hacia su progreso social y además en términos de máxima/relativa/ factible/aceptable eficacia y eficiencia.

Se han realizado más estudios sobre el modo en que el gobierno gobierna a la sociedad, que sobre el modo en que éste se gobierna a sí mismo. Se conoce la estructura de la Administración, pero poco sobre su dirección; conocemos las reglas jurídicas, pero ignoramos los procedimientos y las prácticas que acompañan a las decisiones organizativas. Es decir, sigue existiendo mucha "caja negra" y muchas deficiencias tales como los errores de decisión de los directivos, corrupción, discrecionalidad, fallos en la distribución de la autoridad, en la división del trabajo, en la coordinación y cooperación o intercomunicación en la organización... (Aguilar, 2014:25-26).

\section{3. - EL ESTUDIO DE LAS POLÍTICAS PÚBLICAS}

En la literatura sobre el estudio de las Políticas Públicas se afirma que de los distintos intereses analíticos se derivan las dos grandes vías de investigación que articulan los estudios sobre la materia; Mientras que la más prescriptiva se relaciona con la Ciencia de la Administración y las fases de la formulación y ejecución. La segunda prevalentemente positiva o explicativa tiene a reconstruir las modalidades de los procesos de decisión, las características de los actores y las relaciones entre las distintas fases del proceso de políticas (proceso de políticas) objeto de formular modelos más realistas (Regonini, 1991:62-63) 14. Esta perspectiva última sería resultante de la diferencia entre la ciencia "de" o "para” el proceso de políticas (Policy Making) y basada en la doble acepción implícita del término política (policy), es decir; como actividad valorada en función de los resultados y sus objetivos y; como proceso empírico que exige ser explicado en función de causas y de funciones.

Podría afirmarse pues que mientras el análisis de políticas es la actividad normativa de construir y evaluar la mejor opción de política como solución a determinados problemas públicos, los estudios de políticas (policy studies) se refieren a cualquier estudio relativo a las causas, efectos, procesos, contenido, restricciones, modalidades. . . dela elaboración de la política. Entre ambos extremos tiene lugar toda una serie de posiciones intermedias, lo que explica que son muchos los términos para referirse al conocimiento "de" y "en" la política: policy studies, policy research, policy analysis, policy science (Aguilar, 1992b:69-70) y también, son muchos los intentos de poner orden entre sus diversos significados, acentos, corrientes y polémicas ${ }^{15}$. En definitiva, reflejan la variedad y los criterios y categorizaciones subjetivas ${ }^{16}$, pero permiten el paso a diferentes modelizaciones o enfoques metodológicos como veremos infra.

\section{4. - EL PROCESO DE POLÍTICAS (POLICY MAKING)}

Con el término anglosajón de Policy Making se hace referencia al proceso de formación y elaboración de las políticas entendiéndolo como conjunto de aproximaciones sucesivas a un objetivo deseado frente al cual hay que hacer continuas reconsideraciones. Esta intelección invita a reflexionar sobre el aspecto dinámico del ciclo vital de las políticas. En la secuencia de fases habrá que optar entre el deseo o lo posible, entre lo mejor y el acuerdo o el condicionante argumental de la política misma.

12 El modelo sistémico en la ejecución de las política es deudor de Sharkansky (Sharkansky, 1972)

13 Conferencias Minnowbrook de la Universidad de Siracusa.

14 Vid. en el trabajo citado el interesante mapa conceptual que refleja esta combinación, a la que añade la metodología deductiva vs. inductiva. (Ibídem).

15 El análisis de políticas públicas aparece como una selva de diversas y conflictivas formas de investigación, con terminología inconsistente y estilos intelectuales divergentes e, incluso, con ciertos paradigmas imposibles de comprobar (Torgerson, 1986: 33 ).

16 Para Dye el análisis de políticas puede incluir la investigación de las causas o de las consecuencias de las políticas o programas de gobierno. En los estudios de las causas de las políticas públicas, éstas son las variables dependientes y el analista busca explicarlas con referencia a variables independientes -fuerzas sociales, económicas, tecnológicas, políticas (Dye, 1983: 5-6). 
Para Quade ${ }^{17}$ el análisis de políticas es valioso porque contribuye a la toma de decisiones, ofreciendo información con base en la investigación y el análisis, aislando y esclareciendo las cuestiones, revelando incongruencias en los fines o en los procedimientos, generando nuevas alternativas ysugiriendo las maneras de trasladar las ideas en políticas factibles y viables.

El proceso de políticas implica toda una serie de actividades más o menos relacionadas y no solo es la simple decisión, singular y discreta que se adopta en la toma de la decisión (decission-making), pues ésta atiende sólo el análisis de las intenciones de los responsables que elaboran la política hasta el momento en que deciden la acción gubernamental vinculante, de hacer o no hacer o de hacer de modo diferente. Las consecuencias de la decisión no son entonces objeto de atención en el análisis de la toma de decisiones, mientras que las interacciones y los ajustes continuos que comporta el proceso de políticas hacen que éste sea descrito como proceso más que como una acción única, decidida de una vez por todas (Rose, 1969: 10).

Las ideas que Lindblom expresa en The Policy-Making Process reflejan la tensión entre racionalidad y democracia, entre la eficacia del gobierno y la participación y control de la ciudadanía, tensión que acompaña a cualquier discusión acerca de cómo se elaboran de hecho las políticas o cómo han de elaborarse normativamente. Entre el conflicto entre el análisis y la política en la confección de las políticas hay que optar por la posibilidad de su complementariedad, y si no, hay que optar por la política real y desde esta perspectiva reelaborar el sentido y alcance del análisis. Las interacciones (negociaciones, cooperaciones, intercambios) como actitudes intencionales de solución de problemas colectivos puede substituir al análisis, como políticas pertinentes y eficaces. O son una alternativa al análisis pues por sí mismas frecuentemente resuelven o aminoran los problemas, establecen o hacen las políticas, o logran soluciones en donde falla el análisis.

Pero para Lindblom si bien la interacción política podría sustituir al análisis, también lo necesita, pues a diferencia del análisis racionalista, ajeno a los interjuegos políticos, el análisis se torna como elemento indispensable para las negociaciones políticas, mostrándose como un instrumento de control de los demás en la interacción política y un instrumento de persuasión (Lindblom, 1980:27).

\section{4. - MODELOS ANALÍTICOS}

Las diferentes posiciones en la disciplina influyó en los modelos teóricos de análisis de políticas propuestos ${ }^{18}$ como puede verse en la descripción de síntesis que sigue y que los que nos resultan más relevantes, se exponen a continuación;

\section{1 Modelos incrementalistas (Método rama) ${ }^{19}$}

\subsection{Simple (I)}

- Comparaciones limitadas sucesivas.

- Adecuado para tratar problemas relativamente simples.

- $\quad$ Se parte de la situación actual, paso a paso.

- Análisis limitado a alternativas que difieren sólo incremental y marginalmente respecto del estatus quo.

- Ante consecuencias imprevistas, se puede volver atrás

\section{1. 2 Desarticulado (II)}

- Limitación del análisis a unas pocas alternativas con sus consecuencias.

- Entrelazamiento entre metas y valores con los aspectos empíricos.

- Mayor preocupación analítica por los males a remediar.

- Secuencia de ensayos, errores y corrección de los ensayos.

\section{1. 3 Estratégico (III)}

- Análisis limitado a un conjunto de estrategias calculadas o elegidas.

- Busca simplificar los problemas complejos.

17 Nos advierte enseguida que su denotación del policy analysis es completamente diferente a la del mismo término en el lenguaje de los estudios conductuales del policy making, donde el término se refiere al análisis de los contenidos y de la génesis de las políticas así como de hecho suceden(Quade, 1989).

18 Véase in extenso en Aguilar, 1992 ; 1992b y 1993.

19 Lindblom, 1959, 1963, 1965, 1978, 1980. 
- Recortar el análisis exhaustivo pero buscando un marco teórico.

- Modelo intermedio entre el análisis incremental simple y la racionalidad absoluta.

El incrementalismo I propone actuar directamente en función de los resultados, realizar pequeñas intervenciones, continuar de la misma manera si se consideran positivos los resultados y, en caso de que no se consideren así, retomar el argumento principal y continuar por otro camino.

El incrementalismo II trata de poner a prueba una teoría, observando los resultados como experimentos de un modelo teórico general. Los pasos pequeños son experimentos controlados y sus resultados más fáciles de interpretar.

El incrernentalismo III defiende pasos pequeños, pero justificados por su fácil reversión.

Los modelos deben basarse en el conocimiento teórico del sistema en el que interviene. Sobre una mínima base teórica se pueden recomendar intervenciones incrementales o sinópticas. No hay una clase general de circunstancias que exija sólo cambios pequeños, a veces, es apropiado pensar en grande y otras, en pequeño. (Goodin- Waldner, 1979:9-24).

\section{2 Racionalidad comprensiva (Método raíz/sinóptico/exhaustivo) ${ }^{20}$}

- Parte cada vez de hechos fundamentales nuevos.

- Una decisión es correcta o racional si demuestra que alcanza cierto objetivo.

- Siempre dispuesto a comenzar de nuevo a partir de cero.

- Experiencia como teoría.

- Lo "sinóptico" (como global/completo/exhaustivo) supone el siguiente proceso;

a) Identificar y organizar en una relación coherente la meta y los valores pertinentes para la elección.

b) Identificar todas las alternativas políticas importantes que podrían realizar los valores.

e) Analizar todas las posibles consecuencias importantes de cada una de las alternativas.

d) Elegir la política cuyas consecuencias se acerquen más a los valores pertinentes.

\section{3 Enfoque pluralista/Ajuste partidario mutuo o de intereses ${ }^{21}$}

- El “ajuste mutuo es el mecanismo de coordinación primordial en la decisión, de la cual la negociación es sólo una forma.

- Las decisiones son fragmentadas o descentralizadas y los participantes negocian y se comprometen.

- $\quad$ El acuerdo sustituye al modelo teórico.

- $\quad$ El acuerdo es la prueba de lo correcta.

Lindblom en sus inicios muestra los modelos rama y raíz como antagónicos²2, pero en La Inteligencia de la Democracia" (1965) analiza y etiqueta como nuevo modelo el "ajuste partidario mutuo" 23 y lo presenta como algo distinto del incrementalismo, como teoría aplicable a todas las realidades políticas y en combinación con las otras alternativas, lo que dependerá del estilo de políticas y de la dimensión de su red de políticas²4.

\footnotetext{
20 Lindblom, 1959, 1963, 1965, 1978, 1980.

21 Lindblom, 1959, 1965, 1979.

22 En la Ciencia del salir del paso (Lindblom, 1959).

23 En nota marginal $n^{\circ} 7$ de la obra seminal ya anticiparía que (...)El nexo entre la práctica del método de las comparaciones limitadas sucesivas y los ajustes mutuos de intereses en un proceso de toma de decisiones muy fragmentado añade una nueva faceta a las teorías pluralistas del Gobierno y la Administración (...)

24 El concepto policy networks surge como una forma específica de governance, como reconocimiento de que las políticas emergen de la interacción entre actores públicos y privados, aceptando que el Estado, no es ya el actor dominante en el proceso de políticas y del reconocimiento de la variedad de actores no gubernamentales que participan en los escenarios y en los procesos decisionales (Zurbriggen,
} 2003).

Los actores que participan en la elaboración e implementación de políticas no son solamente los miembros del congreso, de las agencias administrativas y de los grupos de presión, sino también se incluyen expertos, periodistas, analistas políticos, los cuales cumplen un papel importante en la difusión de las ideas políticas. 
Supone que la decisión es descentralizada, con participantes, relativamente autónomos y con cierto pluralismo lo que argumenta un fortalecimiento de la democracia, frente las posiciones dirigistas para resolver mejor las desigualdades ${ }^{25}$.

\section{4 Modelo normativo óptimo ${ }^{26}$}

- Basado en el concepto de innovación.

- Clarificación de valores, objetivos y criterios de decisión.

- Identificación de alternativas y cálculo preliminar de los resultados.

- Consenso entre analistas,

- Incremento del análisis, en función de la información teórica y empírica disponible.

- Mejora del decisión-making desde la experiencia, la iniciativa, la creatividad, el desarrollo de los skills directivos y la promoción del esfuerzo intelectual27.

- Cambio de mentalidad en los enfoques analíticos ${ }^{28}$.

\section{5 Modelos de Allison ${ }^{29}$}

\section{5. 1 Modelo I: Política racional}

- Se escoge la acción que maximiza metas y objetivos

- Los distintos cursos de acción, que se consideran pertinentes para abordar un problema estratégico, configuran la gama de opciones.

- La aprobación de cada opción supone consecuencias que conllevan beneficios y costes respecto de los objetivos estratégicos.

- Se escoge la alternativa de acción cuyas consecuencias favorecen sus metas y objetivos.

\section{5. 2 Modelo II: Proceso organizacional}

- Problemas segmentados y poder fraccionado.

- Los problemas se reparten entre varias organizaciones.

- Las actuaciones se gestionan en subgobiernos.

- Cada organización percibe problemas, procesa información y ejecuta una variedad de acciones casi autónomamente.

- El actor no es ya un todo unitario o monolítico.

\section{5. 3 Modelo III: Política burocrática}

- Las decisiones son fruto de compromisos, coaliciones, competiciones y mal entendidos entre diversos grupos organizativos de las Administraciones.

- Cada grupo es un actor, con un cierto grado de autonomía.

- Se produce un juego competitivo donde el poder tiene un papel importante ${ }^{30}$.

25 Se puede también sostener que el pluralismo no engloba a la totalidad de la sociedad, sino a quienes controlan los canales de expresión.

26 Dror, 1964, 1970: 135-150, 1999:508-520.

27 Dror considera que el alto funcionariado, en la mayoría de los países, se encuentra obsoleto, con bases profesionales inadecuadas y capacidad insuficiente para lidiar con opciones críticas. El autor establece una tipología para caracterizar la evolución del perfil del servicio público, marcando sus fases históricas: a) tipo alpha: (status atribuido, fusión de papeles políticos y administrativos); b) tipo beta: (compra de cargos gubernamentales) y c) tipo gamma: (casi profesionalismo). El tipo delta, se concentraría en las cuestiones de orden estratégico, quedándose las funciones gerenciales para los servidores del tipo gamma y para los servicios técnicos. Esta última alta gerencia -Delta-type senior civil service- debe disponer de unas habilidades especiales, tales como la centralización de las tareas de orden superior, la extremada profesionalización, la innovación y la creatividad, la ética, la autonomía y sentido de trabajo por objetivos (Dror, 1997:5-26).

28 Véase su comparación tentativa entre los rasgos del análisis de sistemas con el análisis político (Dror, 1999:518).

29 Tomados de la experiencia de la crisis de los misiles en Cuba en la Administración Kennedy (Allison, $1969,1971$.

30 Mezcla de las ventajas en la negociación (derivadas de la posición de autoridad y de sus obligaciones formales, del respaldo institucional, de los electores, de la experiencia y del estatus); habilidad y voluntad de usar las ventajas en la negociación; y percepciones que los otros jugadores tienen de los primeros dos componentes. 


\section{6 Enfoque de exploración combinado (Mixed Scanning) ${ }^{31}$}

- Combina elementos de los enfoques racional-comprensivos e incrementalista.

- Utiliza una secuencia de dos enfoques distintos:

a) Gran angular que observaría el todo pero sin gran detalle.

b)Enfoque de aquellas áreas que, descubiertas al inicio, sí necesitan un examen de mayor profundidad.

- A diferencia del incrementalismo, no ignora los problemas y defectos manifiestos de áreas de observación que le son ajenas.

- Estrategia de exploración entre el racionalismo totalitario y el incrementalismo democrático.

\section{7 Análisis de Factibilidad ${ }^{32}$}

- Considera todas las restricciones (policy constraint) relevantes presentes en un contexto de actuación.

- Las restricciones son; sociales, políticas, administrativas, institucionales, técnicas y económicas.

- Las restricciones, restringen durante el proceso decisorio, la gama de elecciones factibles.

- Las restricciones constituyen recursos heurísticos para el aprendizaje, la predicción y la solución de problemas ${ }^{33}$.

\section{8 Análisis como "proceso social" (interacción) ${ }^{34}$}

- El análisis se centra en encontrar un problema sobre el que se deba y pueda hacer algo.

- Los decisores ha de ser capaces de tratar con las variables que tienen bajo su control y en el tiempo disponible.

- Hay que actuar sobre la sociedad para disciplinar el deseo, disminuir las expectativas, ordenar las preferencias.

- Los Gobiernos deben aumentar la productividad y expandir la eficiencia.

- El análisis se muestra como arte.

\section{9 Modelo de papelera/Cubo de basura/Anarquía organizada (Garbage Can) ${ }^{35}$}

- Modelo más opuesto al de maximalismo racional.

- Situación de anarquía organizada, de equivocidad y ambigüedad.

- La decisión es el resultado de la interpretación de muchos flujos en la organización,

- Los flujos giran en torno a; problemas, elecciones, soluciones y actores ${ }^{36}$.

- Los flujos se ven afectados por la estructura organizacional y social.

- Predomina buscar un problema para una solución ya diseñada/adoptada.

- No se no se busca lo óptimo; Se evita lo peor, se gestiona un compromiso, o se sale del apremio ${ }^{37}$.

\section{10 Modelo Teórico Integrado ${ }^{38}$}

- Se considera el análisis como una ciencia de la acción.

- Integra etapas, actores, recursos y productos.

31 Etzioni, 1967.

32 Meltsner, 1972, Majone, 1975.

33 Son productos de decisiones pasadas o de convenciones previas (retroinputs de información).

34 Wildaysky, 1979.

35 Cohen, March, y Olsen, 1972:1-25; Los autores centran su análisis en un tipo de organizaciones que ellos llaman "anarquías organizadas", cuyo referente empírico son las universidades. Kingdon reformula el modelo en los 80 y 90 ofrece un modelo del cubo de basura revisado". Varios autores han identificado a este modelo de toma de decisiones como el de la "irracionalidad" (Olavarría, 2007).

36 La dinámica lógica-racional de los flujos sería; Problema $(\mathrm{P}) \rightarrow$ Elección $(\mathrm{O}) \rightarrow$ Solución $(\mathrm{S}) \rightarrow$ Actores $(\mathrm{A})$.

37 En función de; alternativas privilegiadas (preferidas/predeterminadas/impuestas); experiencias precedentes; tabúes políticos.

38 Subirats y otros, 2010. 
- Posibilita el análisis diacrónico a lo largo de las cuatro etapas.

- El modelo considera todos los factores que puedan contribuir a explicar el buen o mal funcionamiento de las políticas.

- Distingue entre variables a explicar - dimensiones sustantivas e institucionales de los productos y variables explicativas - juego de actores, recursos, y reglas institucionales -

- $\quad$ El modelo ofrece una triple utilidad; descriptiva; analítica (Knowledge of) y prescriptiva (Knowledge for).

- El análisis se focaliza en; el contenido sustantivo, la consolidación del espacio de una política, la consolidación del capital institucional, y la explotación del abanico de recursos.

- Los resultados sustantivos e institucionales en cada etapa tienen una influencia directa sobre el contenido de las subsiguientes.

- Los resultados de cada etapa se ven influenciados por los cambios institucionales y los que operen en los actores o en los recursos.

\section{5. - ¿HETERODOXIA U HETEROPRAXIS?}

El éxito del discurso científico desde los inicios de la disciplina levantó recelos entre algunos de los fundadores, llegando a anunciar el peligro que suponía que personas incompetentes e incluso charlatanas pudieran aprovecharse de una nueva y atrayente etiqueta para legitimar viejos enfoques e investigaciones superficiales ${ }^{39}$.

Las críticas al análisis de políticas se situaron en torno a ser moda pasajera, sustentarse en material teórico obsoleto, ofrecer enfoques demasiado teóricos o demasiado prácticos, muy multidisciplinarios, o muy concentrados en la Ciencia Política, muy cuantitativos, muy subjetivos ideológicamente -liberales o conservadores, deficientes o excesivamente utilizados, ... (Nagel, 1999: 539 y ss). Estas críticas argumentarían en torno a la cuestión fundamental de la objetividad potencial del análisis, en la medida en que éste podría ser realmente objetivo y provisto de metodología pacíficamente aceptada, al menos, para lo más general y universal. Si además también fuera factible, real, útil, posible, pertinente y/o aceptable, no pocas decisiones de hacer, de no hacer o hacer distinto se sustentarían en la viabilidad política o administrativa o resultarían en un fiasco (Nagel, 1999:580).

Las variables analíticas de los modelos expuestos reflejan todo un recorrido entre lo más y lo menos en términos de racionalidad comprensiva, pero estos modelos teóricos no son heterodoxos en sí, pero tampoco son axiomáticos, pues están cargados de problemas epistemológicos y hermenéuticos que hacen dificultosa su aplicación. El relato sobre influencias no es gratuito, pues reflejan como en cualquier marco teórico las influencias de las diversas corrientes del pensamiento predominante de su momento (positivismo, conductismo, ..., con sus revisiones y críticas), las interpretaciones y subjetividades, y por ende, la dificultad de establecer un consenso sobre la idea de lo normativo.

Pensar que la heteropraxis que vivimos (ausencia o análisis precario) es consecuencia de una heterodoxia general en la disciplina no es sostenible, como tampoco que todas las posiciones son ortodoxas como para lograr una fácil ortopraxis. Los puntos siguientes puede ayudarnos a entender el relativismo de todo esto.

\section{1 Conocimiento o política}

En los diferentes enfoques aparecen dos posiciones, que no siendo antagónicas per se, e incluso con aspectos complementarios, sí responden a dos posiciones distintas que condicionaran los modelos. El primer enfoque (sinóptico) es el del "positivismo" 40 , y su principal aspiración es el reemplazo de política por el conocimiento. El conocimiento real era el científico, ligado a la observación de datos, a la inferencia lógica y a la determinación de relaciones constantes entre los hechos. Así los sucesos podrían ser explicados con referencia a condiciones antecedentes y, recíprocamente, los acontecimientos futuros serían susceptibles de ser predichos de manera confiable.

El resultado sería el control de los hechos futuros y, por tanto, podría ser utilizado para desarrollar una tecnología social fiable. El objetivo final analítico ya no sería la computación de estrategias óptimas, sino el diseño de reglas procesales y mecanismos sociales para evaluar evidencia incompleta y frecuentemente contradictoria (Majone, 1977:174).

39 Atribuido a Dror en 1983 (Dror, 1983: 44 y Regonini, 1991:69)

40 Tendencia general en el campo de la filosofía en los 50-60 cuando se buscó ajustar las ciencias sociales al modelo positivista de las ciencias naturales. 
El segundo enfoque (antisinóptico) se enmarca en el "postpositivismo", en el que la política domina al conocimiento. Surge como reacción frente al primer enfoque ya que no deja de ser una tecnocracia irracional resultado de una fuga de la política que deviene en un subrepticio ejercicio del poder ${ }^{41}$.

La primera teoría quedó fascinada en exceso por la razón técnica, olvidando al comportamiento social como factor decisivo, que se incorpora como un elemento activo. El conocimiento ha de ser "de" y "en" la sociedad para lograr su entendimiento cultural. Supone pues un proyecto más participativo, más demócrata y sobre todo, un proyecto social. La razón exige el conocimiento del contexto, orientado a la acción y un análisis que supere las contingencias sociopolíticas en donde se evalúe también el impacto de los efectos colaterales, de tal manera que el juicio humano se vuelva un factor decisivo. Ya no importa tanto si se logra la máxima eficacia y/o eficiencia, sino cuál es la opinión del destinatario.

La lógica de investigación se orienta a la transformación de la vida pública. El conocimiento se extiende hacia lo contextual: es, a la vez, un fin y un medio de la investigación racional y a la par dialógica que terminará en un compromiso político. Hay aquí una revitalización de la política con mayúsculas que, reorientada contextualmente, desmitificando el análisis tecnocrático, y apuntando hacia una dirección post-positivista, pueda constituir una acción política en la que la razón y la política converjan (Torgerson, 1986:33).

Ambas posiciones conllevan roles distintos para los dos jugadores principales, los analistas de políticas y los políticos. A los analistas les interesa la eficiencia económica y cómo las políticas públicas pueden afectar a la sociedad en su conjunto. Lo importante es que los objetivos políticos sean definidos explícitamente y que se jerarquicen los diversos objetivos en competencia. Los analistas quieren medir con claridad los resultados de la política y el grado en que se realizan los objetivos.

A los políticos les interesa el aspecto distributivo de las políticas y su impacto social. Acentúan la negociación y la transacción, variando objetivos si los resultados impiden llegar a un consenso, y resolviendo los problemas en el mismo proceso político. Mientras que el analista es optimista, el político es pesimista, mientras que el analista se preocupa por los costes de oportunidad y opta por el presupuesto por programas, el político opta por objetivos y se ocupa de la distribución (Behn, 1986: 33).

Behn parece mantener una tipología ideal sobre el analista y el político, proponiendo una dicotomía teorética exagerada, pues ambos, como jugadores individuales, poseen una combinación de skills analíticos y políticos. Para Behn, el analista cabal precisa de visión multidisciplinar pasando una y otra vez de lo analítico a lo político y viceversa. El analista debe entender la parte política del juego, de forma que su labor genere la confianza de los decisores políticos e para convertirse en otro miembro del equipo decisor, pues de lo contario el análisis será considerado carente de interés, improductivo e imposible de aplicar, relegado a un mero ejercicio de erudición espuria. Por ello propone hacer análisis más argumentados y más estratégicos y consecuentemente, más convincentes, tendentes a evaluar la factibilidad política de cada alternativa, lo que dependerá de su diseño inteligente, de la estrategia política y de la habilidad en la implementación (Behn, 1986:59).

Vemos pues que el binomio positivismo/ maximalismo analítico puede ser costoso e inconveniente en su aplicación "tout court", para una dinámica que supere el discurso científico, dada la diversidad y los diferentes propósitos de la acción administrativa ${ }^{42}$. Hay que abogar, como regla general, por enfoques que incluyan por una parte racionalidad/ factibilidad en el análisis y, por otra, consenso político y racional al mismo tiempo. Postura acorde con las mejores prescripciones sobre el rol y skills de los analistas (Dror, 1995).

\section{2 Descripción o prescripción}

El intento de conectar el ser con el deber ser, articulando y potenciando una nueva racionalidad que sirva para la prescripción, también está en el debate teórico y ha sido contemplada especialmente ${ }^{43}$ desde las siguientes perspectivas;

1. En Majone podemos encontrar la intuición seminal de que la argumentación puede ser la operación crucial para que el análisis se vuelva política elegida y puesta en práctica. Contrario a la ineficacia de un análisis racionalista de políticas, como ejercicio intelectual orientado a encontrar un racionalismo maximalista, Majone sitúa el análisis en el marco del gobierno democrático, que adopta decisiones mediante la

41 Laswell, que fue tachado de prototipo del positivista/tecnócrata por sus primeras posiciones, terminaría contrarrestando las tendencias oligárquicas y burocráticas para ir convergiendo hacia un método politológico hacia la participación.

42 Pensemos en las diferencias entre actuaciones técnicas y asuntos que afectan al core belief de la sociedad (religión, símbolos, memoria histórica...).

43 Cabe añadir la que ofrece el "Constructivismo", enfocado hacia el problema de los diseños de políticas (Ingram y Schneider, 1997). 
discusión y deliberación pública, resultando central en la política democrática la argumentación. Frente a una estrecha visión de la democracia como transacción de intereses, es más propio la oferta recíproca de razones, los argumentos públicos (Majone, 1989).

En ese marco las decisiones que afectan a la colectividad, en sede democrática, requieren ser explicadas, transmitidas, argumentadas, y merecedoras de una persuasión . por el gobernante que las adopten pensado subjetivamente que es buena. Esto exige la construcción de los argumentos y obtención de evidencias que logren el acuerdo o destierren la resistencia

2. El movimiento Ilamado "Giro Argumentativo (Argumentative Turn) engloba a diferentes enfoques en análisis y planificación de políticas que hacen hincapié en el aumento de la relevancia de la argumentación, el lenguaje y la deliberación en la formulación de políticas. Inspirado por el "giro lingüístico" en el campo de las humanidades, fue desarrollado como una alternativa a las limitaciones epistemológicas de análisis de políticas "neo-positivista” y su comprensión tecnocrática subyacente del proceso de toma de decisiones. El enfoque argumentativo integra sistemáticamente cuestiones empíricas y normativas en un marco metodológico orientado hacia el análisis de la deliberación política. Se muestra muy receptivo al contexto situacional y los múltiples tipos de prácticas de conocimiento involucradas en cada etapa del proceso de la política, Ilamando la atención sobre las diferentes formas de argumentación, persuasión y justificación (Fischer y Forester, 1993).

Igualmente Fischer presenta nuevas perspectivas en el discurso político a partir del análisis discursivo de la política pública y de las prácticas de formulación de políticas públicas deliberativas, como contrapeso a la tecnocracia ${ }^{4}$ dominante y a los enfoques empiricistas de los estudios tradicionales de políticas públicas (Fischer, 2003).

El "Giro Argumentativo revisitado" rechaza la idea de que el análisis de políticas y la planificación son esfuerzos técnicos libres de valores, un enfoque argumentativo tiene en cuenta las formas en que la política se ve afectada por otros factores, como la cultura, el discurso y la emoción. El enfoque revisado se relaciona en particular con los límites de la formulación de políticas racionalistas en el complejo e impredecible mundo de principios del siglo XXI. Hechos como la crisis ambiental, la economía mundial y el desastre de Fukushima hacen de política argumentativa un enfoque urgente para el análisis de políticas y la planificación (Fischer y Gottweis, 2012).

\section{$5 \cdot 3$ ¿El análisis de políticas es el fin de la historia evaluativa?}

Además de las criticas vistas al análisis de políticas han surgido otras propuestas evaluativas en torno a la Nueva Gestión Pública, a las propuestas de Gobernanza, propuestas mixtas o de encuentro45, enfoques contingentes o en torno a perspectivas sistémicas, que escapan ahora de este espacio, pero que invita a pensar sobre la conveniencia o necesidad de buscar modelos complementarios o superadores del análisis de políticas, en cuanto éste puede entenderse como atrapado en un discurso científico que impide, en buena manera, su aplicación en la praxis político administrativa, que de suyo se muestra bastante renuente a su incorporación en la agenda, aun sin conocer su complejidad científica ${ }^{46}$. Pero es inexorable la necesidad de un nuevo devenir científico más acorde a las exigencias ambientales del Cross Modern ${ }^{47}$ caracterizado por una complejidad social creciente originada en la diversidad, la fragmentación y el dinamismo social, al tiempo que se incrementan por el argumento globalizador. Pero la postmodernidad se combina en la actualidad con un proceso de deconstrucción político- administrativa respecto del no puede convertirse en una mera aportación para salir del paso. Es decir, se trata de mejorar el sistema analítico convencional, obteniendo del mismo lo mejor de los modelos normativos, superando a éstos también desde enfoques estratégicos y prospectivos.

Hay que ir considerando ya en la disciplina una suerte de metaanálisis, en clara correspondencia con un metagobierno para otras formas de gobernabilidad como nueva clave de bóveda, y que se corresponda -al mismo tiempo- con variables políticas de mejor democracia en torno a la confianza básica, valor público, legitimación por compromiso y receptividad política, lealtad institucional, innovación... y todo ello, en un entorno de gran complejidad e incertidumbre. En este reto hay cuestiones de mucho calado sin resolver tales como el fracaso de la Nueva Gestión Pública, el metagobierno para la Gobernanza o en la necesidad o no de una Teoría normativa

44 Véase también su aplicación como ruptura de la tecnocracia a favor de propuestas a análisis cualitativos o de variables sociales (Camacho, 2010).

45 (Jiménez, 2008).

46 En ello muy sugerente es la diferenciación entre propósitos abiertos o encubiertos de la evaluación en Gascó, 2002.

47 Término acuñado por Kooiman para referirse al cruce entre lo moderno y lo postmoderno (Kooiman, 2003:60). 
nueva de la Administración Pública sustitutiva del sistema clásico o el futuro del Estado Administrativo y lo niveles supranacionales de decisión y que podrían ayudar a reflexionar sobre el agotamiento del modelo de análisis y en la conveniencia de redefinirlo, superar lo o buscar exploraciones combinadas.

Tarea difícil, sin duda, dado el marco económico e institucional, el desgate del sistema y la infrafinanciación. Sin embargo he aquí el más preciado valor de los políticos y sus asesores: el ingenio necesario para deconstruir y después reedificar, aportando soluciones para los momentos difíciles de aquí y ahora.

\section{6. - DEL DISCURSO A LA PRESCRIPCIÓN NORMATIVA}

En el conjunto de las Administraciones Públicas españolas la situación sobre el análisis de la acción pública podría sustentarse en estas hipótesis;

- Lo predominante es el discurso sobre la praxis evaluativa.

- Este discurso es tanto científico como político.

- La evaluación es fundamentalmente selectiva y excepcional

- La evaluación de la acción pública no es un ejercicio ni habitual ni universal o general.

- La evaluación es más descriptiva que prescriptiva.

Para superar esta situación en España en pro de una dinámica normativa del análisis de la acción pública, hay que indicar que el sentido del término «normativo» que usamos es el empleado en la teoría de decisiones, la Ciencia Administrativa y algunas ramas de la economía. Según el mismo, los analistas deben aspirar a las soluciones óptimas o, por lo menos, a las más eficaces. Pero es cierto que la factibilidad y no lo óptimo, en la mayor parte de ocasiones, es el objetivo realista que debemos plantearnos (Majone, 1975:50). Hay que llegar a la solución factible con renuncia de I o ideal cuando las restricciones contingentes y otras variables de caso sean factores de frustración. Hemos visto que la optimalidad se nutre de las perspectivas científico-positivistas, pero han quedado marginadas por las dominantes posiciones antisinópticas, de ahí que se afirme que si bien el enfoque de políticas es científico, lo es pero en el sentido general de buscar conocimientos verificables, más que en el sentido específico -y casi siempre frustrante y limitante- de buscar leyes generales y aplicables in totum (Ascher, 1986: 365. 389).

Dentro de las características de una Política Pública, se encuentra - junto al contenido, programa, factor de coerción y competencia social - la de “orientación normativa” (Meny-Thoenig, 1992), la cual, supone el propósito de cambio social, de pautas nuevas, positivizadas o institucionalizadas, que serán susceptibles de coerción y/ o compliance ${ }^{48}$ mediante los contenidos constitutivos, regulatorios o distributivos de los programas al efecto según la tipología predominante de políticas (Lowi, 1964, 1972).

En las propuestas seminales de Lasswell el ideal democrático de J. Dewey -como sistema de gobierno y como una forma de vida (Catalán, 2013) - , estuvieron muy presentes e influiría en que Lasswell apareciera como el dirigente intelectual de la integración sistemática del conocimiento y la acción, para lo cual servirían cinco "tareas intelectuales" clave: clarificación de metas, tendencias, condiciones, proyecciones y alternativas. Así el análisis objetivo podía ayudar a efectuar las elecciones fundadas en valores, sin perder por ello su carácter científico, pues su tarea de clarificación de las metas era un ejercicio intelectual.

Los prolegómenos intelectuales previos a la Segunda Guerra Mundial, fueron la base de una infraestructura ideológica en el campo de las políticas que, al cristalizar en los programas del New Deal, aumentó la confianza en la aplicación real de la ciencia social. Para algunos la política era un asunto de valores -ideológico/normativos-, para otros una estrategia, diseño o programa, y para otros abarcaba ambos. Dependiendo de la opción el análisis de políticas es más científico en sus formas, que en la sustancia (Landau, 1977:423-427).

El análisis de políticas es importante si es observado como ciencia social que va a aplicar los resultados de una teoría. Las políticas serán hipótesis, que con sus correspondientes dosis de riesgo e incertidumbre, se presentan para alterar alguna circunstancia o conducta preexistente. En su formulación fijará normativamente la condición de estado buscada y en su implementación, el conjunto de los medios para obtenerla. El objeto de cualquier propuesta de política pretende, al fin, controlar y dirigir cursos futuros de acción. El análisis ha de consistir en el examen de las consecuencias de las políticas públicas ya existentes o de nuevas políticas, en ayudar a establecer los contenidos de nuevas políticas; o en analizar los posibles apoyos de que gozarán las propuestas de nuevas políticas. Y la evaluación posterior en comprobar la consecución de propósitos, labor ex ante y ex post en la que

48 Identificación normativa como asunción voluntaria de la propuesta. 
los valores del marco ideológico-cultural debe ser el principal nutriente.

Entre el maximalismo analítico y la aceptación de que el marco teórico puede ser sustituido por el consenso o que el acuerdo es la prueba de una política "correcta" hay que contemplar otra dinámica normativa que supeditada a la factibilidad, debe de ajustarse para ser comprendida como;

- Continente Ideológico-programático.

- Compromiso político de cambio social innovador.

- Óptimo en términos de búsqueda de máxima racionalidad posible.

- Sinóptico, sistémico u holístico.

- Factible en términos situacionales-contingentes.

- Como superación de la dinámica meramente descriptiva.

Para este propósito hay que erradicar el discurso científico autoafirmatorio, erradicar el discurso político banal y frívolo, y apartarse de una praxis sustentada en el ensayo-error, en el método papelera, o la mera salida del paso, en el mejor de los casos. Todo ello en aras de un nuevo ciclo reformista en la manera de hacer políticas. Precisamos de un bucle reformador que transforme el discurso en realidad, de tal manera que el avance en el momento científico opere en el momento político para que la evaluación de la agenda gubernamental pase del conocimiento "de" al conocimiento "en" o "para". La complejidad social requiere una revolución epistemológica y tecnológica en la acción pública dotada de mayores dosis de inteligencia colectiva y talento para lo público. Hay que apostar por el análisis de la acción pública como instrumento al servicio de la gobernabilidad en el siglo XXI, como la primigenia Ciencia de la Administración -de enfoque predominantemente sociológico - lo fue ante la revolución política y social del Siglo XIX.

\section{BIBLIOGRAFIA}

Aguilar, M. J, -Ander-Egge, E. (1992), Evaluación de servicios y programas sociales, Madrid: Siglo XXI.

Aguilar Villanueva, L. F.

- (1992a), La hechura de las políticas. México: Porrúa.

- (1992b), El estudio de las políticas. México: Porrúa.

- (2014), "Las dimensiones y los niveles de gobernanza", Cuadernos de Gobierno y Administración Pública, $1(1)$.

Alvira, F. (1991), Metodología de la evaluación de programas. Madrid: Centro de Investigaciones Sociológicas.

Allison, G. T.

- (1969), "Conceptual Models and the Cuban Missile Crisis", American Political Science Review, 63(3).

- (1971), Essence of Decision. Boston: Little Brown.

Ascher, W. (1986), "La evolución de las ciencias de políticas: Comprender el surgimiento y evitar la caída", Journal of Policy analysis and Management, 5(2).

Behn, R. D. (1986), “Policy Analysis and Policy Politics”, Policy Sciences. 19.

Bourgon, J. (2007), “Gobierno receptivo, responsable y respetado: Hacia una nueva teoría de la Administración Pública”, Revista Internacional de Ciencias Administrativas, 73-1.

Camacho-Celis, M. (2010), "El giro argumentativo en el análisis de políticas públicas. Una transición desde la tecnocracia a enfoques cualitativos. Aspectos generales sobre la política pública frente al desplazamiento forzado en Colombia", Ponencia para su presentación en el I Congreso GIGAPP. Septiembre, Madrid

Catalán, M. (2013), La Ética de la democracia. Sobre la política de John Dewey. Madrid:Verbum.

Cejudo, G. M. (2008), “Discurso y Políticas Públicas: Enfoque constructivista”, Documentos de trabajo CIDE, 205.

Cohen, M. , March, J. , y Olsen, J. (1972), “A garbage can model of organizational choice”, Journal of Administrative Science Quarterly, 1. 
Dye, T. R.

- (1975). Understanding Public Policy. Englewood Cliffs, NJ: Prentice-Hall.

- (1983). Policy Analysis. The University of Alabama Press.

Dror, Y.

- (1964). Muddling Through, "science" or inertia?", Public Administration Review, 23.

- (1968). Public Policy Making Reexamined. Scranton, Chandler Publishing

- (1970). "Prolegomens to Policy Sciences", Policy Sciences, 1.

- (1983). Public Policy Making Reexamined, $2^{a}$ edic. New Brunswick, Transaction Publishers.

- (1997), “El administrador público tipo Delta para el Siglo XXI, Revista do Serviço Público, Lisboa: ENAP.

- (1999), "Los analistas de políticas: un nuevo papel profesional servicio del gobierno" en Shafritz, J. M. y Hyde, A. C. comps. , Clásicos de la Administración Pública, México: FCE.

- (1995), “Asesores políticos para los Gobiernos”, Documentación Administrativa, 241-242.

Etzioni, A. (1967), “Mixed scanning, a third approach to decision-making”, Public Administration Review, 27.

Fischer, F. y Forester, J. (1993), eds. The Argumentative Turn in Policy Analysis and Planning. Durham: Duke University Press.

Fischer, F. (2003), Reframing Public Policy: Discursive Politics and Deliberative Practices. Oxford University Press.

Fischer, F. , y Gottweis, H. (2012), The Argumentative Turn Revisited: Public Policy as Communicative Practice. Durham: Duke University Press.

Forester, J. (1984). "Bounded Rationality and the Politics of Muddling Through", Public Administration Review, 43.

Frederickson, H. G. (1971), Toward a new public Administration, The Minnowbrook perspective. Chandler publishing Co: Scranton, PA.

García Sánchez, E. (2009), Metaevaluación, Román Reyes (Dir. ), Diccionario Crítico de Ciencias Sociales. Madrid: UCM

Garde Roca, J. A

- (2004), "La evaluación de políticas y su institucionalización en España, Gestión y Análisis de Políticas Públicas, 30-31.

- (2006), "La institucionalización de la evaluación de las políticas públicas en España”, Auditoria Pública, 39.

Garson D. (1986). "De la ciencia de políticas al análisis de políticas: Veinticinco años de progreso”, Análisis de políticas: Perspectivas, Conceptos y Métodos . Grenwich: Jai Press.

Gascó Hernández, M. (2002). "Hacia una sistematización de la evaluación de programas y Políticas Públicas", Gestión de Análisis y Políticas Públicas, 23.

Goodin, R. y Waldner, I. (1979), “Thinking Big, Thinking Small and not Thinking at AH”, Public Policy, 27(1).

Gunn, L. A. (1978), “Why implementation is so difficult?”, Management Services in Government, 163.

Ingram, H. y Schneider, A. (1997), Policy Design for Democracy, Kansas: Univ. of Kansas press.

Ingram, H. (1999), "La implementación una reseña y un marco que se sugiere", Administración Pública. El estado actual de la disciplina, (Lynn, N. B. y Wildavsky, A. , Comps. ). México: FCE.

Jiménez Benítez, W. G. (2008), "El enfoque de políticas públicas y los estudios sobre gobierno. propuestas de encuentro", Reforma y Democracia, 41. Caracas. CLAD.

Kingdon, J. W. (1995), Agendas, Alternatives, and Public Policy. New York: Harper Collins

Kooiman J. (2003) Governing as Governance, London, Sage

Landau, $M$.

- (1972), Political Theory and Political Science. New Jersey: Humanities Press. 
- (1977), “El ámbito propio del análisis de políticas”, American Journal of PoliticalScience, 21(2).

Lasswell, H. D.

- (1951), "The policy orientation”, en Lerner, D. , Lasswell, H. eds, The Policy Sciences. Stanford: University Press.

- (1971), "The Emerging Conception of the Policy Science", Policy Sciences, 1.

Laswell, H-Kaplan, A. (1950), Power and Society: A Framework for Political Inquiry. New Haven: Yale University Pres. Lindblom, C.

- (1959), “The Science of Muddling Through”, Public Administration Review, 19.

- (1963), A Strategy of Decision. New York: Free Press.

- (1965), The Intelligence of Democracy. New York: Free Press.

- (1979), "Still Muddling, Not Yet Through", Public Administration Review, 39(4).

- (1980), The Policy Making Process. Englewood Cliffs: Prentice Hall.

Lowi, T.

- (1964), "American Business and Public Policy Case Studies and Political Theory”, World Polities, 16.

- (1972), "Four Systems of Policy, Politics and Choice”, Public Administration Review, 32.

Majone, G.

- (1975), "La Factibilidad de las políticas sociales", PolicySciences, 6.

- (1989), Evidencia, argumentación y persuasión en la formulación de políticas, New Haven: Yale Univ. Press.

Meny, I. y Thoenig, J. C. (1992), Las Políticas Públicas. Barcelona: Ariel.

Meltsner, J. (1972). "Political Feasibility and Policy Analysis", Public Administration Review, 23.

Nagel, S. (1999), "Evaluaciones opuestas de los estudios de políticas” en Lynn, N. B. y Wildavsky, A. (Comps.) Administración Pública. El estado actual de la disciplina. México: FCE.

Nateras González, M. E. (2006), “Las Políticas Públicas ¿Discurso o realidad?”, Espacios Públicos, 9.

Newton, K, y Sharpe, J. (1984), Does Politics Matter? The determinant of public policy. Oxford: ClarendonPress.

Nora, S. y Minc, A. (1980), La informatización de la sociedad. México: FCE.

Olavarría Gambi, M. (2007), “Conceptos Básicos en el Análisis de Políticas Públicas” Documentos de trabajo, Santiago de Chile: INAP-Instituto de Asuntos Públicos. Universidad de Chile.

Perry, J. y Kraemer, J. (1983), Public management: Public and Private perspectives. California: Mayfield Publishing Co.

Pinazo Hernandis, J.

- (2007), Neoinstitucionalismo Estratégico y Gobernabilidad: Un enfoque sistémico y prospectivo para la Gestión Pública en América latina. Alicante: Univ. Alicante.

- (2013), Administración Pública y Gobernabilidad. De los orígenes a los nuevos paradigmas. Valencia: Lowcostbooks.

Pressman, J. L. y Wildavski, A. (1973), Implementation. Berkeley: Univ. California Press .

Quade, E. S. (1989), Análisis de formación de decisiones públicas, Madrid: Instituto de Estudios Fiscales.

Regonini, G. (1991). "El estudio de las Políticas Públicas”. "Políticas Públicas y Organización administrativa”, Documentación Administrativa, 224-225.

Rein, M. y Rabinovitz. F. (1978), "Implementation: A Theoretical Perspective”, en W. D. Burhamy M. W. y Weinberg, eds. , American Politics and Public Policy. Cambridge: The MIT Press. IEP.

Royo-Vilanova S. (1961), "La Función Pública", La Administración Pública y el Estado contemporáneo. Madrid: 
Rose, R. (1969), Policy-making in in Britain, London: Macmillan.

Sabatier, P. A. y Mazmanian, D. A. (1981). The Implementation of Public Policy: A Framework of Analysis", Effective Policy Implementation. Lexington, Ma: Lexington Books.

Sharkanski, I.

- (1972) "The Analysis of public policy: recent additions to an ancient and honorable literature", Midwest Journal of Political Science, 16.

- (1999), “Lo que un politólogo puede decir a un político acerca de la probabilidad de éxito o fracaso", en Shafritz, J. M. y Hyde, A. C. comps, Clásicos de la Administración Pública. México, FCE.

Smith, T. B. (1973) “The Policy implementation process”, Policy Sciences, 3.

Subirats Humet, J. (1994), Análisis de Políticas Públicas y eficacia de la Administración. Madrid: MAP.

Subirats Humet, J. y otros (2010), Análisis y Gestión de Políticas Públicas. Barcelona: Ariel.

Torgerson, D. (1986), “Entre el conocimiento y la política: tres caras del análisis de políticas”, PolicySciences, 19.

Urwick, L. (1961), Los elementos de la Administración. Londres: Pitman\&Sons.

Van Meter, D. y Van Horn, C. (1975) “The Policy Implementation Process. A conceptual Framework", Administration \& Society, 6(4).

Wildaysky, A. (1979), Speaking Truth to Power. Boston: Little Brown.

Zurbriggen, C. (2003), “Las redes de políticas públicas. Una revisión teórica”, Colección Documentos del Institut Internacional de Governabilitat de Catalunya, Barcelona, 